\title{
Bisphenol A and Bisphenol S Oxidative Effects in Sheep Red Blood Cells: An In Vitro Study
}

\author{
E. Baralla, M. P. Demontis, M. V. Varoni, and V. Pasciu \\ Department of Veterinary Medicine, University of Sassari, Sassari, Italy \\ Correspondence should be addressed to V. Pasciu; vpasciu@uniss.it
}

Received 30 December 2020; Revised 4 March 2021; Accepted 11 March 2021; Published 25 March 2021

Academic Editor: Fatma M. El-Demerdash

Copyright @ 2021 E. Baralla et al. This is an open access article distributed under the Creative Commons Attribution License, which permits unrestricted use, distribution, and reproduction in any medium, provided the original work is properly cited.

\begin{abstract}
Bisphenols (BPs) are plastic components widely used worldwide and occurring in the environment. Exposure to these compounds is known to be harmful for animals and humans at different levels. The aim of this study was to evaluate and compare the oxidative effects of bisphenol A (BPA) and bisphenol S (BPS) in sheep. Reactive oxygen species (ROS) production and correlated structural alterations in sheep erythrocytes were investigated in vitro. Blood samples from four ewes were collected at fasting from the jugular vein using vacuum collection tubes containing EDTA. For ROS assay in erythrocytes, blood was properly diluted and BPA or BPS was added to obtain final bisphenol concentrations in the range between 1 and $300 \mu \mathrm{M} .2^{\prime}, 7^{\prime}$-Dichlorodihydrofluorescein diacetate (H2DCF-DA) $3 \mu \mathrm{M}$ was added to the samples, and fluorescence was read in four replicates using a microplate reader. To evaluate erythrocyte shape, blood smears of blood treated with the different concentrations of BPS and BPA were prepared. A significant increase in ROS production was observed when concentrations of BPS and BPA increased from 1 to $100 \mu \mathrm{M}(p<0.05)$. At the higher concentrations of the two studied BPs $(300 \mu \mathrm{M}$ of BPS and 200-300 $\mu \mathrm{M}$ of BPA), a ROS decrease was observed when compared to the control group $(p<0.01)$. Erythrocytes' shape alterations were observed in cells treated with BPS and BPA 200$300 \mu \mathrm{M} 4$ hours after the beginning of the treatment. This study confirms that BPA and BPS exhibit oxidative effects on sheep erythrocytes. At higher concentrations, BPA was able to modify erythrocytes' shape, while BPS altered their membrane as a sign of a protein clustering that could lead to eryptosis. These BPs' effects are consequent to intracellular ROS increase.
\end{abstract}

\section{Introduction}

Bisphenol A (BPA) is an endocrine disruptor that has been widely used in the world with several uses like in the manufacturing of plastics, epoxy resins, and a variety of plastic and paper consumer products and food [1-3]. There are many concerns about its well-known endocrine disrupting action as well as about its hepatotoxic, neurotoxic, and carcinogenic effects on the human body. Several studies report that these BPA toxic effects are also associated with the production of ROS $[4,5]$, and sometimes, antioxidants' use can prevent these effects [6]. A significant increase in the levels of intracellular ROS production and a decrease of antioxidant capacity was found in KGN cells (a granulosa-like tumor cell line) after treatment with high concentrations of BPA and its analogues [7]. BPA and the products of its metabolism can reach the body and, accordingly, the blood, through the assumption of drinking water and food and through dental material [8-12]. Due to the toxicity of this compound, and to comply with restrictions and regulations, manufacturers have progressively replaced it with substitutes. One of the main substitutes used is bisphenol S (BPS). However, BPS has become so common in society that it was determined in urine samples of $81 \%$ of people in the United States and Asia [13]. Therefore, over the last few years, there has been a considerable scientific effort to evaluate the safety of BPS, since most people and animals are exposed to it through different routes. Unfortunately, several studies report that BPS has similar androgenic/estrogenic effects in different experimental models $[14,15]$.

The extended use of BPs has led to high environmental exposure of these compounds [16]. Moreover, given the widespread use of BPs, they are ubiquitous in the environment, and their accumulation can be dangerous not only for humans but also for animals that, through different routes, can come in contact with them. Plastics are in fact widely used in 
agriculture and animal breeding for a variety of purposes like covering for crops and wrappings for hay bales.

BPA and its analogues can come into interaction with plasma proteins, hemocytes, and, first of all, with erythrocytes as carriers for xenobiotics. Being the most plentiful cells in the circulatory system, erythrocytes are responsible for transporting oxygen and other chemicals; thus, they are strongly exposed to xenobiotics like bisphenols (BPs) [12]. Maćczak et al. (2015) reported that BPA, bisphenol $\mathrm{F}$ (BPF), BPS, and bisphenol AF (BPAF) exhibited different hemolytic and oxidative potentials and caused morphological changes in red blood human cells.

The aim of our study was to investigate the effects of BPs in sheep through an in vitro study in ewes' erythrocytes. To avoid pain and permanent danger to animals, the in vitro approach permits to predict risks related to the environment and animals together with the application of the 3Rs principle, Reduce, Refine, Replace animal use, in accordance with the Directive 2010/63 EU. The sheep model represents a valuable tool in studying the effects of xenobiotics on animals and humans also at different development stages [17]. As an example, sheep was used to help understand BPs' effects and mechanism of action in a pregnancy model [18-20]. Another author used sheep blood for his work, and in particular, he studied sheep blood contaminated with BPA that was detected in both plasma and red blood cells (RBC) (10 times lower than in plasma), indicating that BPA could have migrated from plasma into RBC [21]. Guignard et al. (2016) reported that part of the BPA ingested by sheep could be directly absorbed by passive diffusion through the buccal mucosa due to the molecule's physicochemical properties [22]. In this way, BPA directly attained the systemic circulation avoiding the hepatic first-pass effect. Furthermore, sheep thoroughly masticates its food, thus ensuring a long enough contact of the contaminated food with the buccal mucosa. In this animal model, a dual mechanism of absorption was observed. In fact, the mucosal absorption during food chewing was followed by the one from the gastrointestinal tract. This dual mechanism might therefore be associated with higher bioavailability and higher blood concentrations than routes of administration limited to intestinal absorption as reported by pharmacokinetic studies $[23,24]$.

Environmental contaminants can interfere with physiological systems and ruminants' capacity to reproduce and fight diseases, especially when animals are grazed on contaminated pastures or drinking water. In light of this, understanding the effects of pollutants requires knowledge at the cellular and molecular levels.

Given these premises, in this work, we report a preliminary study to evaluate the oxidative effect produced by BPs on sheep erythrocytes. In particular, we compared the effect of BPA and BPS on ROS production and related structural alterations in vitro in the cellular model described.

\section{Materials and Methods}

All procedures involving animals in this study were approved by the Local Animal Care and Use Committee (authorization code: 2899 of 17/01/2018). Ewes were confined outdoors with access to a sheltered area, at the experimental facilities of the Department of Veterinary Medicine at the University of Sassari, Italy $\left(40^{\circ} 43^{\prime} 40.33^{\prime \prime} \mathrm{N}, 8^{\circ} 33^{\prime} 1.33^{\prime \prime} \mathrm{E}\right)$. Blood samples from four healthy ewes were collected at fasting (07:00 a.m.) from the jugular vein using $10 \mathrm{~mL}$ vacuum collection tubes containing EDTA K2 (Vacutainer Systems Europe; Becton Dickinson, Meylan Cedex, France). A pool of blood was used for the analysis. The mean concentration of sheep erythrocytes was $12 * 10^{6}$ cells $/ \mu \mathrm{L}$.

Blood was diluted 1:50 with Hanks' Balanced Salt Solution (HBSS) and used for the treatment with BPA and BPS for hemolysis test and for blood smear preparation. For ROS assay, a further dilution was made as described below. BPA and BPS stock solutions were prepared in methanol.

2.1. ROS Assay. For the measurement of ROS production in erythrocytes, blood was further diluted 1:4 using HBSS (total dilution $1: 400$ ), adding BPA or BPS at different concentrations $(1,10,100,200$, and $300 \mu \mathrm{M})$ and $2^{\prime}, 7^{\prime}$-dichlorodihydrofluorescein diacetate $\left(\mathrm{H}_{2} \mathrm{DCF}-\mathrm{DA}\right)$ at the final concentration of $3 \mu \mathrm{M}$. Within the cell, the esterases cleave the acetate groups on $\mathrm{H}_{2}$ DCF-DA, thus trapping the reduced form of the probe $2^{\prime}, 7^{\prime}$-dichlorodihydrofluorescein $\left(\mathrm{H}_{2} \mathrm{DCF}\right)$. Intracellular ROS oxidize H2DCF, yielding the fluorescent product, DCF. Fluorescence was measured using a FLUOstar Omega microplate reader (BMG LABTECH). Excitation and emission wavelengths used for fluorescence quantification were 485 and $535 \mathrm{~nm}$, respectively. All fluorescence measurements $(\mathrm{RFU}=$ Relative Fluorescence Unit) were corrected for background fluorescence. Treatments for 4 hours with BPs induced variation of fluorescence versus control (CTRL) (cells without BPs treated with methanol) and were measured in four replicates. Data were expressed as means $\pm \operatorname{SD}[25,26]$.

2.2. Preparation of Blood Smears. Diluted blood was incubated at $37^{\circ} \mathrm{C}$ for 4 hours with different concentrations of $\operatorname{BPS}$ and $\operatorname{BPA}(1,10,100,200$, and $300 \mu \mathrm{M})$. After incubation, blood samples were used to prepare blood smears. A slide for each concentration of BP was viewed at high magnification using an inverted system microscope (Olympus X71 model TH4200). An untreated blood smear was used as the control.

2.3. Hemolysis. After incubation at $37^{\circ} \mathrm{C}$ for 4 hours with BPA and BPS concentrations ranging from 1 to $300 \mu \mathrm{M}$, blood samples were gently centrifuged ( $100 \mathrm{~g}$ for $3 \mathrm{~min}$ ), and the supernatant was used for hemolysis test. The absorbance of the hemoglobin in the supernatant was read at $405 \mathrm{~nm}$. Data were expressed as $\%$ means \pm DS. The absorbance value in the supernatant of sheep $\mathrm{RBC}$ lysed in $\mathrm{H}_{2} \mathrm{O}$ was considered as $100 \%$ hemolysis [27].

2.4. Statistical Analysis. Statistical Analysis Data are expressed as mean \pm SEM of at least four replicates. Results were analyzed by a monofactorial ANOVA (Minitab ${ }^{\circledR} 18.1$ ). Statistical significance was accepted at $p<0.05$.

\section{Results}

3.1. ROS Assay. ROS production in sheep erythrocytes treated for 4 hours with BPA and BPS at concentrations ranging from 


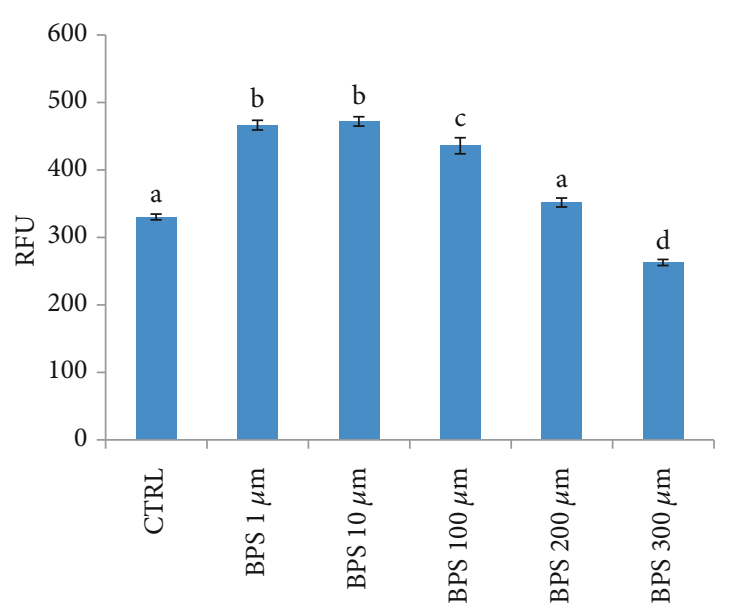

(a)

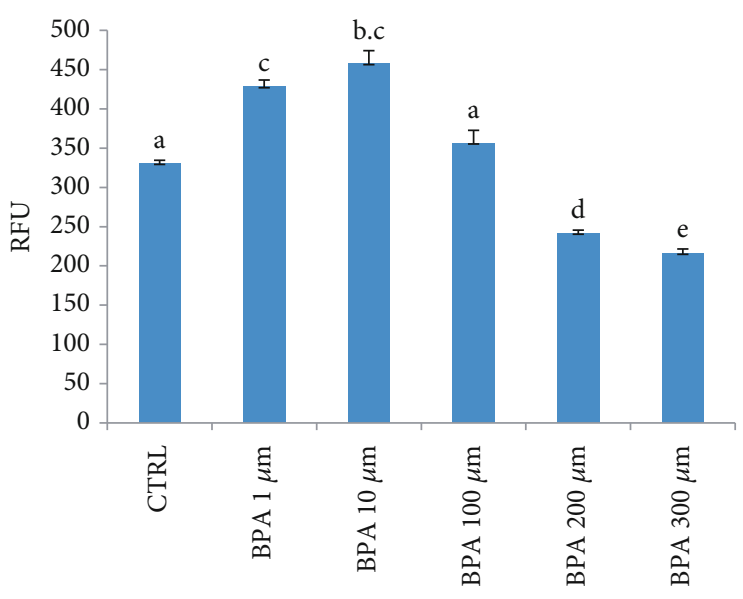

(b)

FIGURE 1: ROS production in sheep erythrocytes treated with BPS (a) and BPA (b) (1-300 $\mu \mathrm{M})$. All fluorescence measurements (RFU) after 4 hours of treatment were corrected for background fluorescence, and excitation and emission wavelengths were 485 and $535 \mathrm{~nm}$, respectively. CTRL were cells treated with methanol. Statistical Analysis Data are expressed as mean \pm SEM of at least 4 replicates. Results were analyzed by a monofactorial ANOVA (Minitab ${ }^{\circledR} 18.1$ ). Statistical significance was accepted at $p<0.05$.

$1 \mu \mathrm{M}$ to $300 \mu \mathrm{M}$ was determined. As shown in Figure 1 , a significant increase in ROS production was observed when concentrations of BPS and BPA increased from 1 to $100 \mu \mathrm{M}$ $(p<0.01$ for 1 and $10 \mu \mathrm{M}$ and $p<0.05$ for $100 \mu \mathrm{M})$ (Figure $1(\mathrm{a})$ ) and from 1 to $10 \mu \mathrm{M}(p<0.01$ for $1 \mu \mathrm{M}$ and $p$ $<0.05$ for $10 \mu \mathrm{M}$ ) (Figure 1(b)), respectively, when compared to the control group. Furthermore, at the higher concentrations of the two studied BPs $(300 \mu \mathrm{M}$ of BPS and 200$300 \mu \mathrm{M}$ of BPA), a ROS decrease was observed when compared to the control group (Table 1). In RBC treated with BPS at the concentration of $200 \mu \mathrm{M}$ (Figure 1(a)) and BPA at the concentration of $100 \mu \mathrm{M}$ (Figure 1(b)), ROS production was reported to values similar to that of the control group.

3.2. Blood Smears. Blood smears of erythrocytes treated for 4 hours with the tested BP concentrations were performed. They were read in order to evaluate possible structural changes in the shape of RBC when compared to blood smears of erythrocytes of the control group.

Erythrocytes' shape alterations were observed in cells treated with BPS and BPA 200-300 $\mu \mathrm{M} 4$ hours after the beginning of the treatment (Figure 2). RBC treated for 4 hours with other concentrations of BPs investigated in this work did not show any shape alteration.

3.3. Hemolysis. After incubation or sheep RBC with BPs, a significant hemolysis increase was observed with increasing BPA and BPS concentrations (from 100 to $300 \mu \mathrm{M})(p<0.01$; Figure 3), with a major hemolysis \% observed in RBC treated with BPA.

\section{Discussion}

BPs' wide use has led them to become ubiquitous in the environment, causing several harmful effects for human and animal health. Furthermore, the exposure of humans and animals to these chemicals can be chronic, from the prenatal
TABLE 1: \% in ROS production in erythrocytes increases significantly when compared to control. Statistical Analysis Data are expressed as mean \pm SEM of at least 4 replicates. Results were analyzed by a monofactorial ANOVA (Minitab ${ }^{\circledR} 18.1$ ).

\begin{tabular}{lcc}
\hline Bisphenols & $\begin{array}{c}\text { Bisphenols } \\
\text { concentrations }\end{array}$ & $\begin{array}{c}\text { \% ROS production vs. } \\
\text { CTRL }\end{array}$ \\
\hline $1 \mu \mathrm{M}$ & $+29.2 \pm 7.1 \uparrow^{* *}$ \\
& $10 \mu \mathrm{M}$ & $+30 \pm 6.9 \uparrow^{* *}$ \\
BPS & $100 \mu \mathrm{M}$ & $+24.2 \pm 11.9 \uparrow^{*}$ \\
& $200 \mu \mathrm{M}$ & $+6 \pm 6.5$ \\
& $300 \mu \mathrm{M}$ & $\downarrow^{* *}-20 \pm 4.49$ \\
\hline & $1 \mu \mathrm{M}$ & $+22.8 \pm 8.8 \uparrow^{* *}$ \\
BPA & $10 \mu \mathrm{M}$ & $+28^{*} \pm 16.8 \uparrow^{*}$ \\
& $100 \mu \mathrm{M}$ & $+7 \pm 5$ \\
& $200 \mu \mathrm{M}$ & $\downarrow^{* *}-26.9 \pm 4.22$ \\
& $300 \mu \mathrm{M}$ & $\downarrow^{* *}-34.65 \pm 5.82$ \\
\hline
\end{tabular}

$\uparrow^{*}$ statistically significant increment $(p<0.05)$; $\uparrow^{* *}$ statistically significant increment $(p<0.01)$; $\downarrow^{* *}$ statistically significant decrement $(p<0.01)$.

stage to adulthood. BPs are indeed constituents of daily used products, and the contact with them can be either indirect or direct [28]. The study of the effects of chemicals such as BPs can be favorably developed on erythrocytes, given that RBC are relatively easy to obtain, can be used for in vitro or in vivo experiments, and are sensitive biomarkers of chemical-induced damage. Maćczak et al. $(2015,2017)$ investigated for the first time the effects of BPA and some of its analogues on the oxidative stress system in human erythrocytes, confirming their ability to induce oxidative changes in this nonnucleated cells, also if they are considered to be more resistant to oxidative stress than nucleated ones. In another study, the same authors reported that BPA and its 


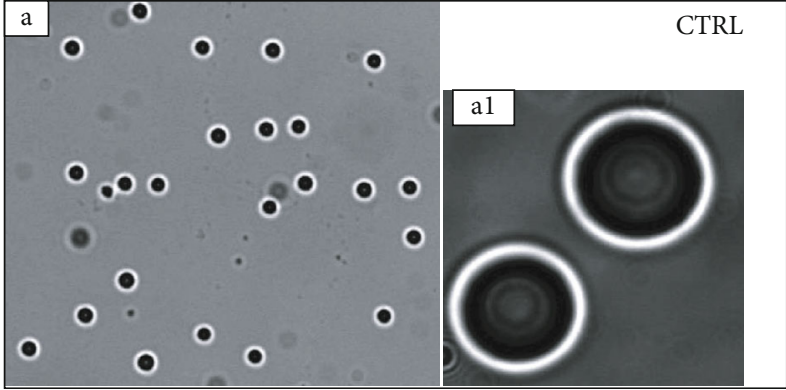

(a)

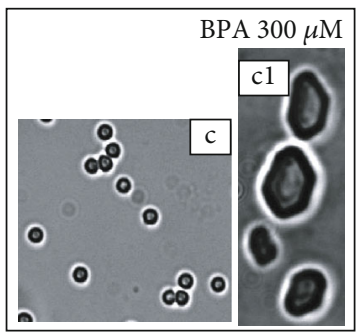

(c)

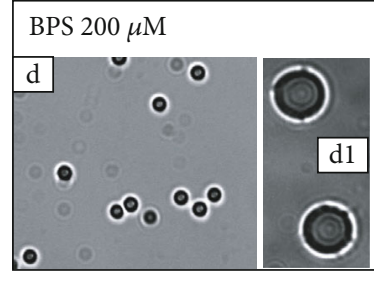

(d)

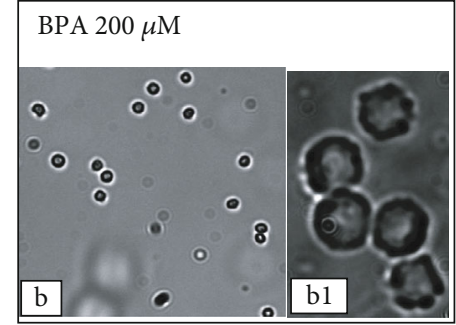

(b)

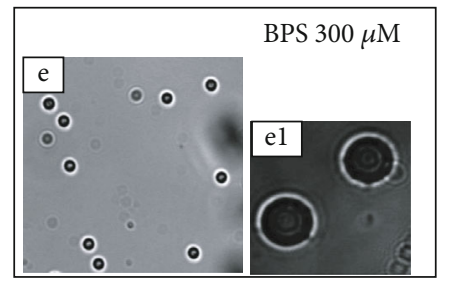

(e)

FIGURE 2: Smears of sheep's blood: shape of erythrocytes treated with methanol (CTRL) ((a) 10x and (a1) 100x) with BPA 200 $\mu$ M ((b) 10x and (b1) 100x), BPA $300 \mu \mathrm{M}$ ((c) 10x and (c1) 100x), BPS $200 \mu \mathrm{M}$ ((d) 10x and (d1) 100x), and BPS 300 $\mu \mathrm{M}$ ((e) 10x and (e1) 100x), 4 hours after the beginning of the treatment.

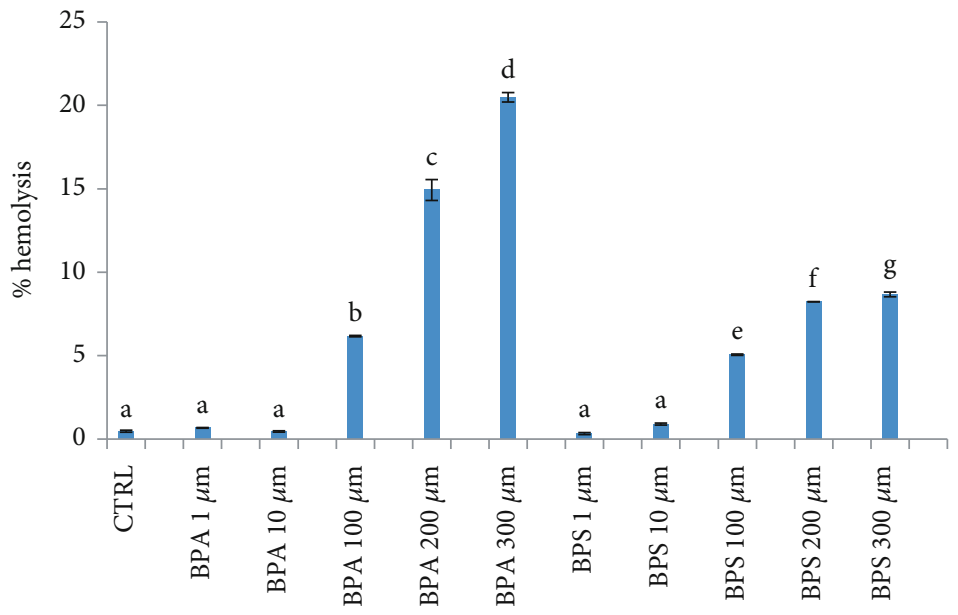

FIGURE 3: RBC hemolysis percentage after incubation with BPA and BPS at concentrations of 1, 10, 100, 200, and 300 $\mu$ M; CTRL were cells treated with methanol. Statistical Analysis Data are expressed as mean \pm SEM of at least 4 replicates. Results were analyzed by a monofactorial ANOVA (Minitab ${ }^{\circledR}$ 18.1). Lowercase letters indicate significant differences between groups $(p<0.01)$.

analogues caused changes in cytosolic calcium ion levels in human erythrocytes after 4 hours of treatment [12]. Other literature data revealed that eryptotic changes are associated with the increase of intracellular calcium ions in RBC. Moreover, it was found that eryptosis was triggered by $\mathrm{H}_{2} \mathrm{O}_{2}$ and paralleled by increased ROS and $\left[\mathrm{Ca}^{2+}\right]$ and that erythropoietin, which possesses antioxidant property, could protect erythrocytes against oxidative stress-induced eryptosis [29]. It was also reported that alterations in intracellular ROS caused eryptosis, along with other modifications like alterations of calcium ion levels, caspase-3, calpain activation, and phosphatidylserine translocation in this cell type [30].
At the moment, no study is reported in the literature investigating the oxidative effects of BPs in sheep erythrocytes. Nevertheless, sheep is an excellent animal model to study several physiological or pathological conditions [18]. Moreover, given the high accumulation in the environment of plastic products [31], livestock can be directly exposed to many emerging contaminants like BPs. Furthermore, direct oral absorption of BPs during food chewing in the buccal mucosa, might be associated with higher bioavailability and higher blood concentrations of this chemical in sheep [22].

In this ruminant, exposure to BPs can also occur though drinking water, contaminated food, and ingestion of soil. 
Moreover, animal feed and water can be stored in plastic containers that, with the help of the high temperatures reached in certain seasons, can contribute to the release of BPs, representing a threat for these domestic ruminants.

In this work, we studied ROS production in vitro in sheep erythrocytes, treated with different BPA and BPS concentrations $(1-300 \mu \mathrm{M})$ for 4 hours. We observed an increase in ROS production at increasing concentrations of BPA (1$10 \mu \mathrm{M})$ and $\operatorname{BPS}(1,10,100 \mu \mathrm{M})$. At the higher concentrations of the two studied BPs (200 and $300 \mu \mathrm{M}$ of BPA and $300 \mu \mathrm{M}$ of BPS), ROS production decreased when compared to control (Figure 1). This decreasing condition could be explained probably because the oxidative damage was so great as to trigger hemolysis, which in vivo can result in eryptosis, as reported in the literature $[12,29,30]$. This aspect was confirmed by the observed alteration of erythrocytes treated with BPS and BPA 200-300 $\mu \mathrm{M} 4$ hours after the beginning of the treatment (Figure 2). Membrane erythrocytes' alteration observed in this study after treatment with BPA at the highest concentration used confirms results obtained by Maćczak et al. (2015). The same authors did not observe any RBC shape's modification when cells were treated with BPS at concentrations also higher than those used in our study. Nevertheless, in this work, erythrocytes treated with BPS $300 \mu \mathrm{M}$ did not show any shape modification in line with Maćczak et al. (2015), but as shown in Figure 2, alterations in membrane integrity were observed.

Several studies report that the accumulation of oxidative damage in erythrocytes leads to irreversible clustering of integral membrane protein band 3. This clustering is an essential process of RBC senescence and eryptosis that can result in cellular removal [32-35].

Under in vitro conditions, the damaged erythrocytes cannot be eliminated and they undergo hemolysis [27] as observed in our study (Figure 3). In particular, high BPA concentrations caused a higher $\%$ of hemolysis when compared to its analogue BPS. This trend is in accordance with what the above described for shape and membrane integrity RBC alterations.

Many studies evaluate several harmful effects of BPS [14, 36-38] like oxidative stress, and results found in this study are in line with them. Indeed, our results confirm that, also if BPS is considered as a safer alternative to BPA, it is able to exhibit oxidative effects on sheep erythrocytes also if to a lesser extent than its regulated analogue as reported also by Maćczak et al. (2017) for human erythrocytes.

\section{Conclusions}

At the moment, BPs' impact studies are relatively few in sheep, and results obtained in this work show that BPs are able to exert oxidative effects in erythrocytes of this livestock animal. Given that BPs are ubiquitous in the environment and have a wide range of toxicity profiles, these findings highlight the potential adverse effects of BP exposure which should require further interest from competent authorities.

\section{Data Availability}

Data used to support the findings of this study are included within the article.

\section{Conflicts of Interest}

The authors declare that there is no conflict of interest regarding the publication of this paper.

\section{References}

[1] C. Liao, F. Liu, H.-B. Moon, N. Yamashita, S. Yun, and K. Kannan, "Bisphenol analogues in sediments from industrialized areas in the United States, Japan, and Korea: spatial and temporal distributions," Environmental Science \& Technology, vol. 46, no. 21, pp. 11558-11565, 2012.

[2] C. Liao and K. Kannan, "Concentrations and profiles of bisphenol A and other bisphenol analogues in foodstuffs from the United States and their implications for human exposure," Journal of Agricultural and Food Chemistry, vol. 61, no. 19, pp. 4655-4662, 2013.

[3] A. M. Molina, N. Abril, N. Morales-Prieto et al., "Evaluation of toxicological endpoints in female zebrafish after bisphenol A exposure," Food and Chemical Toxicology, vol. 112, pp. 1925, 2018.

[4] S. Lee, K. Suk, I. K. Kim et al., "Signaling pathways of bisphenol A-induced apoptosis in hippocampal neuronal cells: role of calcium-induced reactive oxygen species, mitogen-activated protein kinases, and nuclear factor-kappaB," Journal of Neuroscience Research, vol. 86, no. 13, pp. 2932-2942, 2008.

[5] J. I. Eid, S. M. Eissa, and A. A. El-Ghor, "Bisphenol A induces oxidative stress and DNA damage in hepatic tissue of female rat offspring," The Journal of Basic \& Applied Zoology, vol. 71, pp. 10-19, 2015.

[6] H. G. Abdel-Rahman, H. M. A. Abdelrazek, D. W. Zeidan, R. M. Mohamed, and A. M. Abdelazim, "Lycopene: hepatoprotective and antioxidant effects toward bisphenol Ainduced toxicity in female Wistar rats," Oxidative Medicine and Cellular Longevity, vol. 2018, Article ID 5167524, 8 pages, 2018.

[7] M. Huang, S. Liu, L. Fu, X. Jiang, and M. Yang, "Bisphenol A and its analogues bisphenol $\mathrm{S}$, bisphenol $\mathrm{F}$ and bisphenol AF induce oxidative stress and biomacromolecular damage in human granulosa KGN cells," Chemosphere, vol. 253, p. $126707,2020$.

[8] K. Makarova, P. Siudem, K. Zawada, and J. Kurkowiak, "Screening of toxic effects of bisphenol A and products of its degradation: zebrafish (Danio rerio) embryo test and molecular docking," Zebrafish, vol. 13, no. 5, pp. 466-474, 2016.

[9] H. Terasaka, Y. Kadoma, H. Sakagami, and S. Fujisawa, "Cytotoxicity and apoptosis-inducing activity of bisphenol A and hydroquinone in HL-60 cells," Anticancer Research, vol. 25, no. 3b, pp. 2241-2247, 2005.

[10] R. M. Nachman, J. C. Hartle, P. S. J. Lees, and J. D. Groopman, "Early life metabolism of bisphenol A: a systematic review of the literature," Current Environmental Health Reports, vol. 1, no. 1, pp. 90-100, 2014.

[11] A. Maćczak, B. Bukowska, and J. Michałowicz, "Comparative study of the effect of BPA and its selected analogues on hemoglobin oxidation, morphological alterations and hemolytic changes in human erythrocytes," Comparative Biochemistry and Physiology Part C: Toxicology \& Pharmacology, vol. 176177, pp. 62-70, 2015.

[12] A. Maćczak, M. Cyrkler, B. Bukowska, and J. Michałowicz, "Eryptosis-inducing activity of bisphenol $\mathrm{A}$ and its analogs in 
human red blood cells (in vitro study)," Journal of Hazardous Materials, vol. 307, pp. 328-335, 2016.

[13] C. Liao, F. Liu, H. Alomirah et al., "Bisphenol S in urine from the United States and seven Asian countries: occurrence and human exposures," Environmental Science \& Technology, vol. 46, no. 12, pp. 6860-6866, 2012.

[14] A. Ullah, M. Pirzada, S. Jahan, H. Ullah, and M. J. Khan, "Bisphenol A analogues bisphenol B, bisphenol F, and bisphenol S induce oxidative stress, disrupt daily sperm production, and damage DNA in rat spermatozoa: a comparative in vitro and in vivo study," Toxicology and Industrial Health, vol. 35, no. 4, pp. 294-303, 2019.

[15] S. Eladak, T. Grisin, D. Moison et al., "A new chapter in the bisphenol A story: bisphenol S and bisphenol $\mathrm{F}$ are not safe alternatives to this compound," Fertility and Sterility, vol. 103, no. 1, pp. 11-21, 2015.

[16] A. Mendy, P. M. Salo, J. Wilkerson et al., "Association of urinary levels of bisphenols $\mathrm{F}$ and $\mathrm{S}$ used as bisphenol A substitutes with asthma and hay fever outcomes," Environmental Research, vol. 183, p. 108944, 2020.

[17] R. C. Cardoso and V. Padmanabhan, "Prenatal steroids and metabolic dysfunction: lessons from sheep," Annual Review of Animal Biosciences, vol. 7, no. 1, pp. 337-360, 2019.

[18] J. Gingrich, Y. Pu, R. Ehrhardt, R. Karthikraj, K. Kannan, and A. Veiga-Lopez, "Toxicokinetics of bisphenol A, bisphenol S, and bisphenol $\mathrm{F}$ in a pregnancy sheep model," Chemosphere, vol. 220, pp. 185-194, 2019.

[19] M. Puttabyatappa, J. D. Martin, V. Andriessen et al., "Developmental programming: changes in mediators of insulin sensitivity in prenatal bisphenol A-treated female sheep," Reproductive Toxicology, vol. 85, pp. 110-122, 2019.

[20] O. Teteau, M. Jaubert, A. Desmarchais et al., "Bisphenol A and S impaired ovine granulosa cell steroidogenesis," Reproduction, vol. 159, no. 5, pp. 571-583, 2020.

[21] J. Sajiki, "Simple and accurate determination of bisphenol A in red blood cells prepared with basic glycine buffer using liquid chromatography-electrochemical detection," Journal of Chromatography B, vol. 783, no. 2, pp. 367-375, 2003.

[22] D. Guignard, G. Gauderat, V. Gayrard et al., "Characterization of the contribution of buccal absorption to internal exposure to bisphenol A through the diet," Food and Chemical Toxicology, vol. 93, pp. 82-88, 2016.

[23] T. Corbel, V. Gayrard, C. Viguié et al., "Bisphenol A disposition in the sheep maternal-placental-fetal unit: mechanisms determining fetal internal exposure," Biology of Reproduction, vol. 89, no. 1, p. 11, 2013.

[24] G. Gauderat, N. Picard-Hagen, P. L. Toutain et al., "Bisphenol A glucuronide deconjugation is a determining factor of fetal exposure to bisphenol A," Environment International, vol. 86, pp. 52-59, 2016.

[25] V. Pasciu, E. Baralla, M. V. Varoni, and M. P. Demontis, "Evaluation of curcuma and ginger mixture ability to prevent ROS production induced by bisphenol S: an in vitro study," Drug and Chemical Toxicology, pp. 1-7, 2019.

[26] S. Waris, A. Patel, A. Ali, and R. Mahmood, "Acetaldehydeinduced oxidative modifications and morphological changes in isolated human erythrocytes: an in vitro study," Environmental Science and Pollution Research International, vol. 27, no. 14, pp. 16268-16281, 2020.

[27] M. Mischitelli, M. Jemaà, M. Almasry, C. Faggio, and F. Lang, "Ca2+ entry, oxidative stress, ceramide and suicidal erythro- cyte death following diosgenin treatment," Cellular Physiology and Biochemistry, vol. 39, no. 4, pp. 1626-1637, 2016.

[28] J.-J. Kim, S. Kumar, V. Kumar, Y.-M. Lee, Y.-S. Kim, and V. Kumar, "Bisphenols as a legacy pollutant, and their effects on organ vulnerability," International Journal of Environmental Research and Public Health, vol. 17, no. 1, p. 112, 2020.

[29] Y. Sun, G. Liu, Y. Jiang, H. Wang, H. Xiao, and G. Guan, "Erythropoietin protects erythrocytes against oxidative stress-induced eryptosis in vitro," Clinical Laboratory, vol. 64, no. 3, pp. 365-369, 2018.

[30] M. Jarosiewicz, J. Michałowicz, and B. Bukowska, "In vitro assessment of eryptotic potential of tetrabromobisphenol A and other bromophenolic flame retardants," Chemosphere, vol. 215, pp. 404-412, 2019.

[31] R. Geyer, J. R. Jambeck, and K. L. Law, "Production, use, and fate of all plastics ever made," Science Advances, vol. 3, no. 7, pp. e1700782-e1700782, 2017.

[32] P. Arese, F. Turrini, and E. Schwarzer, "Band 3/complementmediated recognition and removal of normally senescent and pathological human erythrocytes," Cellular Physiology and Biochemistry, vol. 16, no. 4-6, pp. 133-146, 2005.

[33] M. M. Kay, K. Sorensen, P. Wong, and P. Bolton, “Antigenicity, storage, and aging: physiologic autoantibodies to cell membrane and serum proteins and the senescent cell antigen," Molecular and Cellular Biochemistry, vol. 49, no. 2, pp. 6585, 1982.

[34] A. Pantaleo, G. Giribaldi, F. Mannu, P. Arese, and F. Turrini, "Naturally occurring anti-band 3 antibodies and red blood cell removal under physiological and pathological conditions," Autoimmunity Reviews, vol. 7, no. 6, pp. 457-462, 2008.

[35] H. Shimo, S. N. V. Arjunan, H. Machiyama et al., "Particle simulation of oxidation induced band 3 clustering in human erythrocytes," PLOS Computational Biology, vol. 11, no. 6, p. e1004210, 2015.

[36] A. Maćczak, M. Cyrkler, B. Bukowska, and J. Michałowicz, "Bisphenol A, bisphenol S, bisphenol $\mathrm{F}$ and bisphenol AF induce different oxidative stress and damage in human red blood cells (in vitro study)," Toxicology In Vitro, vol. 41, pp. 143-149, 2017.

[37] X. Xiao, X. Zhang, C. Zhang et al., "Toxicity and multigenerational effects of bisphenol S exposure to Caenorhabditis elegans on developmental, biochemical, reproductive and oxidative stress," Toxicology Research, vol. 8, no. 5, pp. 630640, 2019.

[38] C. Xie, M. Ge, J. Jin et al., "Mechanism investigation on bisphenol S-induced oxidative stress and inflammation in murine RAW264.7 cells: the role of NLRP3 inflammasome, TLR4, Nrf2 and MAPK," Journal of Hazardous Materials, vol. 394, p. $122549,2020$. 